\title{
Introduction to the Special Issue: "Families in Canada: Diverse, Complex, and Evolving"
}

\author{
Nathan Battams ${ }^{1} \cdot$ Nora Spinks $^{1} \cdot$ Sara MacNaull $^{1}$
}

Published online: 25 March 2020

(C) Springer Nature Switzerland AG 2020

Families in Canada are diverse, complex, and unique - a cornerstone of society and the engine of our economy. Families are society's most adaptable institution, continuously evolving across generations as family members adjust and react to social, economic, cultural, and environmental forces while managing their multiple responsibilities. Over the years, a robust and growing body of research has highlighted many important contributions that families make to the well-being of individuals, workplaces, communities, and society.

Since the Vanier Institute of the Family was founded in 1965, we have collaborated and partnered with those who study, serve, and support families to strengthen the national understanding of families and family life. But what does this mean, and how is it done?

Just as "it takes a village to raise a child," we have worked with a growing and interdisciplinary network of professionals and practitioners; academics and scholars; and organizations and governments to generate rigorous and reliable research, create and share accessible resources for learning about families, and facilitate evidence-based discussion and decision-making. With this community of minds and talents, we analyze data, synthesize research, and mobilize knowledge to support evidence-informed decisions, evidence-based policies and programs, and evidence-inspired innovation to optimize family well-being.

We are honored to have been asked by Simona Bignami, editor of Canadian Studies in Population, to serve as guest editor for this special issue. We have curated a wide range of interconnected and complimentary papers related to families and family life. This collection highlights some of the work being done by researchers from several disciplines across the country, many of whom were involved with our Families in Canada Conference events last year.

Each of the papers makes a unique contribution to Canadian research on families, family life, and family experiences. Some tackle long-standing or recently recognized data gaps, which result from methodological challenges and the ever-changing

Sara MacNaull

smacnaull@vanierinstitute.ca

1 The Vanier Institute of the Family, Ottawa, Canada 
landscape of available information. Some provide nuance and clarity, highlighting the rich diversity inherent in family life while recognizing shared or common realities. All of the papers make distinct and valuable contributions to conversations taking place on a variety of topics, including work and family, family well-being, research methodologies, and data gaps. Our focus is as much on how we learn about families as it is on what we have learned.

In their study "Does Living Alone Mean Spending Time Differently? Time Use and Living Arrangements among Older Canadians," Xiangnan Chai and Rachel Margolis examine the social, mental, and physical well-being of the growing numbers of seniors living alone. The authors examine data from the 2015 General Social Survey on Time Use focused on behaviors related to healthy and active aging, such as communication and connection with others, nutrition and physical activity, self-care, and caregiving.

Military family well-being is examined in "Intra-Provincial Variation in Publicly Funded Mental Health and Addictions Services Use Among Canadian Armed Forces Families Posted Across Ontario." In this piece, lead author Isabel Garcés Davila and her colleagues make innovative use of administrative data to shed light on the impact of the high mobility associated with military life on the family's access to - and usage of mental health and addictions services.

Canadian research on coupling (i.e., marriage, living common-law) and uncoupling (i.e., separation, divorce) has been limited since national data on marriage and divorce has not been tracked since 2008. The resulting data gap has had an impact on what we know about families and family trends over the past decade, as well as research into the impact of these transitions. In "The Growing and Shifting Divorced Population in Canada," Rachel Margolis and Youjin Choi use Census and population estimate data to provide a portrait the current divorced population, measure trends over time, and assess the well-being of this population.

While it is not a new phenomenon, multi-partner fertility (having children with more than one partner) has not been thoroughly studied in the Canadian context - a knowledge gap resulting primarily from methodological challenges involved with identifying and tracking complex family behaviors. In "Regional Variations in Multiple-Partner Fertility in Canada," Ana Fostik and Céline Le Bourdais take on this challenge, using Census data to explore and document this family experience, including regional disparities in the family trajectories that lead to this family pathway.

In "Canadian Employers' Reaction and Policy Adaptation to the Extended, 61-Week Parental Leave," Rachael Pettigrew explores the impact of changes to employment insurance (EI) policy implemented in 2017, which provides parents with the option of up to 18 months of EI benefits leave with smaller monthly benefit payments. In providing the employer's perspective on the impact of this policy, her study provides new insights into organizational culture and employees' work-life responsibilities and how employers and employees have reacted to this policy.

Kerri Claire Neil and Barbara Neis continue the work-family conversation in “"Mobility Has Always Been a Part of My Life': Work-related Mobility and Families in Canada." In this research note, the authors provide an overview of the key findings from the On the Move Partnership - a multi-year research program investigating employment-related geographical mobility, which includes new approaches to filling data gaps and knowledge mobilization to tell the stories behind the statistics. Touching on how mobility affects military families, temporary foreign workers, workers with 
long-distance commuting, and more, Neil and Neis highlight the diversity among mobility experiences and the multifaceted impacts it has on workers, families, employers, and communities from coast to coast to coast.

Finally, in their research note "Indigenous Families and Households in Canada: A Tale of Statistical Disadvantage," Simona Bignami-Van Assche and Charles-Olivier Simard provide important insights into research on First Nations, Métis, and Inuit families in Canada, which has been limited by a lack of data on the composition of Indigenous households and how they have evolved over time. As published Census reports have not included so far household-level indicators disaggregated by Indigenous identity, there is little quantitative data on household change. As the authors explain, "statistical representation" of Indigenous peoples in population research is essential to building a shared future for all families. This will be increasingly important moving forward as First Nations principles of OCAP ${ }^{\circledR}$ (ownership, control, access, and possession) provide Indigenous communities with greater control over data collection processes in their communities and how this information can be used.

As we approach the 30th anniversary of the United Nations International Year of the Family in 2024 and work toward achieving the Sustainable Development Goals (SDGs), collaboration across disciplines, sectors, and geographies will be increasingly important to national and international conversations about family well-being. We thank the editors, authors, and reviewers for their valuable contribution to the journal. The next step will be to mobilize this knowledge, continue this research, share these insights, foster innovation, facilitate conversations, and expedite research to practice to optimize family well-being in Canada and around the world. 